\title{
Estudio de fatiga y fractura de monocristales, bicristales y policristales de la aleación Cu-18,2 Zn-7,0 Al con memoria de forma ${ }^{(\bullet)}$
}

\author{
F.X. Gil ${ }^{(*)}$ y J.Me. Guilemany ${ }^{(* *)}$ \\ Resumen Se estudian las propiedades mecánicas de tracción, compresión y resistencia a la fatiga de \\ monocristales, bicristales y policristales de la aleación $\mathrm{Cu} 18,2 \% \mathrm{Zn}-7,0 \%$ Al. Se relaciona su \\ comportamiento mecánico con la microestructura del material ensayado. \\ Palabras clave: Memoria de forma. Fatiga. Fractura.

\section{Study of fatigue and fracture of poly-, bi- and single-crystals of $\mathrm{Cu}-18.2 \mathrm{Zn}-7.0 \mathrm{Al}$ shape memory alloys}

\begin{abstract}
Mechanical properties -tensile, compression and fatigue stresses- of poli-, bi- and single-crystals of $\mathrm{Cu}-18.2 \mathrm{Zn-7.0} \mathrm{Al}$ have been studied. The relationship between mechanical behaviour and microstructure has been determined.
\end{abstract}

Keywords: Shape memory. Fatigue. Fracture.

\section{INTRODUCCIÓN}

Desde el punto de vista de sus aplicaciones tecnológicas, las aleaciones policristalinas en fase $\beta$ de $\mathrm{CuAlNi}, \mathrm{CuZnAl}$, e incluso CuAlMn, presentan un serio problema a causa de la fractura intergranular (1-3). Por otra parte, las aleaciones Ni-Ti se deforman de manera dúctil aproximadamente un $50 \%$ (45). S. Miyazaki y col. (6) sugieren que la causa de la fractura intergranular en aleaciones de base cobre con fase $B$ es la concentración de tensiones en los límites de grano, debido a la elevada anisotropía elástica y al considerable tamaño de grano de estas aleaciones. La gran ductilidad de las aleaciones Ni-Ti

(•) Trabajo recibido el día 11 de noviembre de 1994.

${ }^{*}$ Dpto. Ciencia de los Materiales e Ingeniería Metalúrgica. E.T.S. Ingenieros Industriales de Barcelona. Universidad Politécnica de Cataluña. Avda. Diagonal, 647. 08028Barcelona (España).

(**) Dpto. Ingeniería Química y Metalurgia. Ciencia de los Materiales y Metalurgia Física. Facultad de Química. Universidad de Barcelona. c/ Martí i Franqués, 1. 08028Barcelona (España). se debe a la escasa anisotropía elástica y al pequeño tamaño de grano.

En las aleaciones con memoria de forma de base cobre policristalinas con fase $B$, se observa que la rotura se inicia y se propaga a lo largo del límite de grano, mientras que si la estructura es martensítica la propagación de la rotura se desvía fácilmente desde los límites de grano a la matriz, ya que las deformaciones son asimiladas por las placas martensíticas para producir otras variantes menos favorables (movimiento de placas) que producen un relajamiento en los límites de grano.

K. Takezawa y col. (7) estudiaron la fractura intergranular en aleaciones $\mathrm{Cu}-\mathrm{Zn}-\mathrm{Al}, 4 \%$ at $\mathrm{Al}$ y $31,6 \%$ at $\mathrm{Zn}$ y concentración electrónica $e / a=1,4$. Para ello, se realizaron ensayos de tracción en monocristales, bicristales y policristales. En los ensayos con monocristales se obtuvieron grandes deformaciones, próximas al $40 \%$. En los realizados en un bicristal con el límite de grano paralelo a la dirección de la tensión, se alcanzó una deformación del $60 \%$ hasta la rotura. Estos valores de deformación son mayores que en monocristal, debido a que las placas martensíticas se inducen homogéneamente y sus 
deslizamientos son uniformes en sus dos direcciones. Cuando el límite de grano es perpendicular a la tensión aplicada, estas deformaciones no se alcanzan, ya que en este caso la deformación no es homogénea.

En policristales de esta misma aleación, la deformación hasta la rotura es menor de la mitad que en el caso del monocristal.

M. Sade y cols. (8-10) han estudiado la fatiga en monocristales de $\mathrm{Cu}-\mathrm{Zn}-\mathrm{Al}$ controlando la carga a diferentes frecuencias. Mediante microscopía electrónica de transmisión, observaron como las dislocaciones son un obstáculo para la transformación martensítica. También, apreciaron la poca influencia que tienen la composición química y la orientación cristalina en la vida a fatiga del material.

\section{MATERIAL Y MÉTODO EXPERIMENTAL}

Se estudiaron las propiedades mecánicas de tracción, compresión y fatiga en nueve muestras de la aleación $\mathrm{Cu} 18,21 \mathrm{Zn}-6,99 \mathrm{Al}$ (\% en masa). La composición química de las muestras se analizó por absorción atómica y microanálisis EDS, resultando tener todas las muestras idéntica composición. Las temperaturas de transformación de estas muestras se determinaron mediante un calorímetro de flujo, resultando ser las que ofrece la tabla I. La estructura metalográfica de colada de la aleación se muestra en la figura 1, en la que se aprecia la fase $\alpha$ y el eutectoide formado por las fases $\alpha$ y $\gamma$. Las muestras, antes de su estudio, se sometieron a un tratamiento térmico a $850{ }^{\circ} \mathrm{C}$ durante $30 \mathrm{~min}$ y posteriormente a un temple en agua a $25^{\circ} \mathrm{C}$. La microestructura resultante después del tratamiento térmico es fase $\beta$, responsable de la pseudoelasticidad que presenta la aleación.

De las nueve muestras estudiadas, tres corresponden a policristales obtenidos en horno de resistencias por fusión del metal encapsulado en cuarzo al vacío (11). El tamaño de grano de las muestras es de 170 $\mu \mathrm{m}$ de diámetro con una morfología equiaxial. Estos valores se determinaron mediante técnicas de análisis de imagen (12).

TABLA I- Temperaturas de transformación, ${ }^{\circ} \mathrm{C}$

TABLE I-Transformation temperatures, ${ }^{\circ} \mathrm{C}$

\begin{tabular}{|l|c|c|c|c|}
\hline & $M_{\mathrm{s}}$ & $M_{\mathrm{f}}$ & $A_{\mathrm{s}}$ & $A_{\mathrm{f}}$ \\
\hline Policristal & -9 & -36 & -22 & -2 \\
\hline Monocristal & -9 & -32 & -22 & 18 \\
\hline $\begin{array}{l}\text { Bicristal } \\
(311) / /(111)\end{array}$ & -8 & -34 & -20 & 10 \\
\hline $\begin{array}{l}\text { Bicristal } \\
(311) / /(110)\end{array}$ & -6 & -30 & -19 & 12 \\
\hline
\end{tabular}

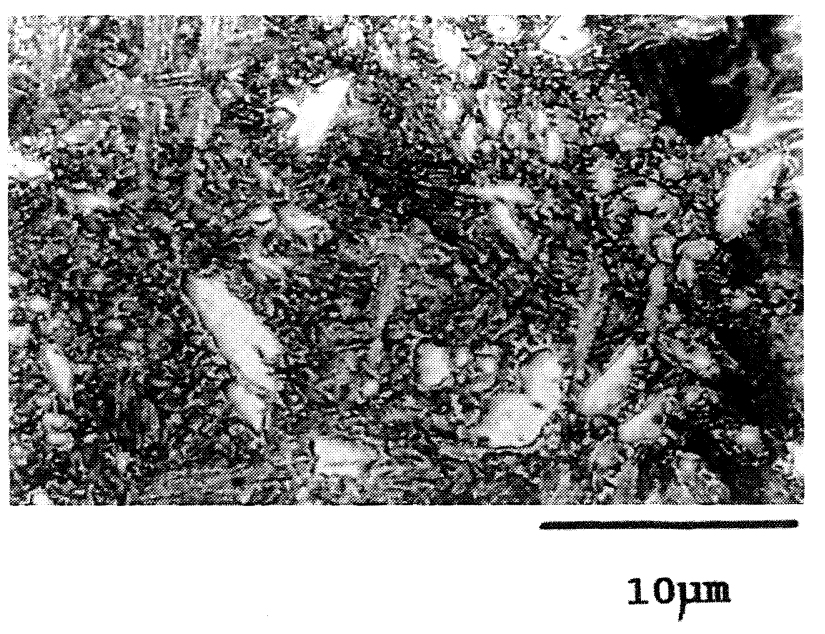

FIG. 1. - Estructura de colada.

Fig. 1.-As-cast structure.

Otras tres muestras corresponden a monocristales producidos mediante el método de Bridgman (13), con orientaciones cristalográficas iguales (311). Los bicristales se obtuvieron por el mismo método; uno de ellos, con orientaciones de (311) y (110), y los otros dos con orientaciones cristalográficas (311) y (111).

Las muestras con las que se realizaron los ensayos de compresión eran de forma cilíndrica, y sus medidas $h=6,5 \mathrm{~mm}$ y diámetro $=5 \mathrm{~mm}$. Las probetas de tracción y fatiga se mecanizaron siguiendo la norma ASTM E8M, con posterior pulido de la superficie. La mecanización de las probetas se realizó con la microestructura de colada, ya que en este caso su mecanizado es mejor. Una vez obtenidas las probetas, se llevaron a cabo los tratamientos térmicos para conseguir la fase $\beta$ y eliminar la acritud que la mecanización hubiera podido producir, según se ha comentado anteriormente.

Los ensayos se realizaron en una máquina servohidráulica INSTRON, aplicando una velocidad de movimiento de mordazas de $10 \mathrm{~mm} / \mathrm{min}$ para los ensayos de compresión y de tracción.

En los ensayos de fatiga se controló la deformación, con una velocidad de la misma de $6,5 \times 10^{-3}$ $s^{-1}$ y $R=-1$; la amplitud de deformación estudiada fue de $5 \times 10^{-3}$, llevándose las probetas hasta rotura. Los ensayos de fatiga se llevaron a cabo sobre probetas pulidas especularmente, para que el acabado superficial no influyera en la vida a fatiga del material.

Los bicristales ensayados tenían el límite de grano perpendicular al eje de tensión.

\section{RESULTADOS EXPERIMENTALES Y DISCUSIÓN}

Los resultados de los ensayos de tracción y compresión se muestran en la tabla II, que ofrece la 
TABLA II- Tensiones de transformación $(\beta \rightarrow$ Martensita inducida por tensión), carga máxima y deformación hasta la rotura a partir de ensayos de tracción y compresión en policristales, monocristales y bicristales

TABLE II- Transformations stresses $(\beta \rightarrow$ stressinduced martensite), maximum load and deformation to rupture from tensile and compression test in poli- mono- and bi-crystals

TRACCIÓN

\begin{tabular}{|l|c|c|c|}
\hline & $\sigma_{\mathrm{B} \rightarrow \mathrm{M}}, \mathrm{MPa}$ & $\sigma_{\max }, \mathrm{Mpa}$ & $\epsilon, \%$ \\
\hline Policristal & 90 & 406 & 18 \\
\hline $\begin{array}{l}\text { Monocristal } \\
(311)\end{array}$ & 116 & 208 & 38 \\
\hline $\begin{array}{l}\text { Bicristal } \\
(311) / /(111)\end{array}$ & 102 & 290 & 12 \\
\hline
\end{tabular}

COMPRESIÓN

\begin{tabular}{|l|c|c|c|}
\hline Policristal & 70 & 425 & 6 \\
\hline $\begin{array}{l}\text { Monocristal } \\
(311)\end{array}$ & 98 & 310 & 25 \\
\hline $\begin{array}{l}\text { Bicristal } \\
(311) / /(111)\end{array}$ & 85 & 360 & 10 \\
\hline
\end{tabular}

tensión de transformación, la tensión máxima y la deformación hasta la rotura.

De los resultados de la tabla II se deduce que:

- Tanto en los ensayos de tracción como en los de compresión, la tensión de transformación $\beta \rightarrow$ SIM es mínima para el policristal y máxima para el monocristal.

Esto se debe a dos causas; en primer lugar, en el policristal, al contener granos con diferentes orientaciones, siempre hay una que favorece en mayor medida la transformación martensítica inducida por tensión; es decir, una orientación, con un factor de Schmid más favorable a la transformación y que, por tanto, precisará una tensión menor para la nucleación de las primeras placas de martensita. Una vez nucleadas estas primeras placas, se crea un campo de tensiones alrededor de las de tipo elástico que provocará su propagación por el interior del grano.

Asimismo, el hecho de que en el policristal la transformación martensítica inducida por tensión (SIM) ocurra a tensiones menores, se debe al efecto de los límites de grano en la transformación martensítica. Los límites de grano favorecen la nucleación de SIM, ya que tienen una tensión interna que propicia la nucleación en límites de grano de placas martensíticas así como su propagación (14), según se deduce de la figura 2 , en la que se observan pla-

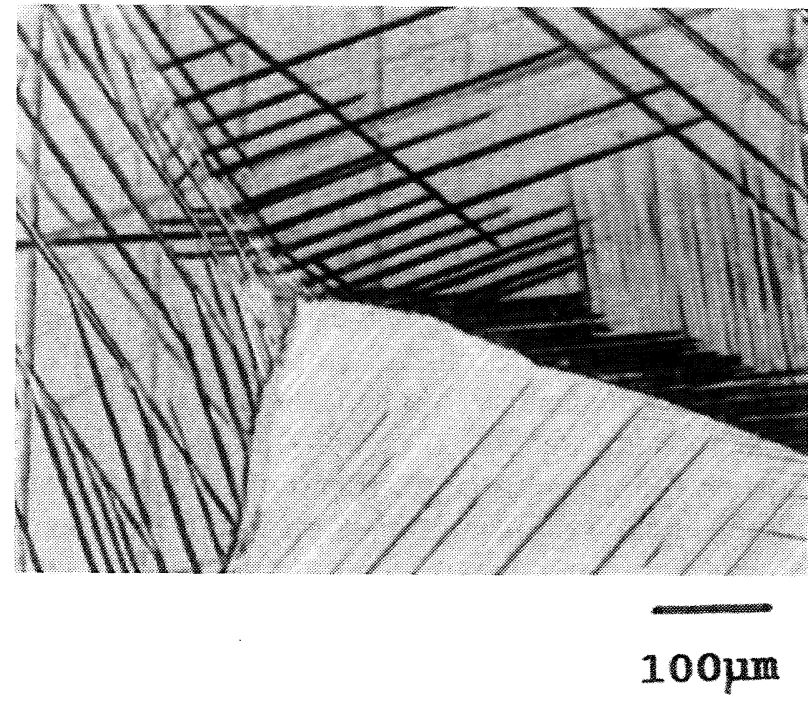

FIG. 2.- Placas martensíticas en los límites de grano.

Fig. 2.-Martensite plates in grain boundaries.

cas de martensita que crecen a partir de los límites de grano. Este hecho se observa más claramente en las aleaciones con memoria de forma de base cobre con respecto a las de $\mathrm{Ni}-\mathrm{Ti}$, ya que en aquéllas la anisotropía es mayor y, por tanto, la tensión interna en los límites de grano es superior.

Además, en trabajos anteriores (15) se observó mediante microscopía electrónica de barrido que en las placas martensíticas, cuando su crecimiento llega al límite del cristal, se produce una nucleación y una rápida propagación en el grano contiguo que tiene una orientación cristalográfica menos favorable para la transformación martensítica. Ello se debe a que la interacción de la placa con el límite de grano produce una energía elástica que al sumarse a la interna propia del límite de grano, produce energía suficiente para vencer la tensión crítica de transformación y provocar la aparición de martensita en dicho grano. La etapa de nucleación de las primeras placas martensíticas es crítica en este tipo de transformaciones, ya que desencadenan la transformación en todos los granos del policristal; este hecho se constata en los registros calorimétricos, observándose que los termogramas son de tipo burst, es decir, que se produce un cambio muy brusco en la señal diferencial al iniciarse la transformación y una pequeña histéresis entre las temperaturas singulares de transformación.

- Los monocristales son menos resistentes, pero mucho más dúctiles, debido a que la ausencia de límites de grano proporciona mayor movilidad a las dislocaciones. Además, los monocristales no presentan incompatibilidades en la deformación, que se producen en los límites de grano de los policristales y que están relacionadas con la 
anisotropía de la deformación propia de las variantes de martensita. Esto se corrobora en las fractografías de los policristales y bicristales estudiadas por MEB donde se observa fractura frágil, apreciándose por el contrario en los monocristales fractura dúctil con grandes áreas de deformación plástica. La figura 3 muestra una fractura intergranular del policristal ensayado a tracción, apreciándose los diferentes granos sin deformación plástica.

- Los bicristales con el límite de grano perpendicular al eje de tensión se comportan de manera más parecida a la de los policristales que a la de los monocristales. En todos los casos, se observa la rotura intergranular. En estos bicristales, denominados asimétricos, se produce deformación heterogénea y la deformación hasta la rotura es mucho menor que en monocristales. En el caso de los bicristales asimétricos, con el límite de grano paralelo a la dirección de la tensión, la deformación puede ilegar a alcanzar el $60 \%$ hasta rotura (7). Estos valores de deformación son mayores que en el monocristal, hecho que se debe a que las placas martensíticas se inducen homogéneamente y los deslizamientos son uniformes en las dos direcciones. Esta homogeneidad produce una coherencia de placas entre los dos cristales.

- Al realizar el ensayo de compresión, las tensiones de transformación $B \rightarrow$ SIM son menores que en el ensayo de tracción; es decir, con esfuerzos compresivos es más fácil inducir martensita.

Este hecho se debe a la triaxialidad de esfuerzos en el ensayo de compresión, que provoca transfor-



$100 \mu \mathrm{m}$

FIG. 3.- Fractura intergranular de un policristal sometido a tracción.

Fig. 3.- Intergranular fracture in a policrystal subjected to tensile stresses maciones en diferentes orientaciones cristalinas de la aleación. En el caso del ensayo de tracción, una variante de martensita es mucho más preferente que las demás dependiendo del eje de tensión y, por tanto, es la que se formará. Esta selección de variante por parte del material exigirá un nivel superior de tensión mecánica. En la figura 4 se aprecia la morfología de las placas martensíticas producidas por enfriamiento brusco; como puede observarse, dichas placas tienen orientaciones diferentes que se denominan autoacomodadas, ya que adquieren disposiciones en las que la energía necesaria para su formación es mínima. Como contraste, la figura 5 muestra la morfología de las placas martensíticas inducidas por tensión; en este caso, se puede observar cómo las orientaciones en las que se disponen son preferenciales según el eje de tensión y la orientación del cristal.

\subsection{Comportamiento frente a la fatiga}

Los ensayos de deformación cíclica requieren la aplicación de tensiones de $138 \mathrm{MPa}, 132 \mathrm{MPa}$ y $126 \mathrm{MPa}$ en el primer ciclo para policristales, bicristales y monocristales, respectivamente, y que, por tanto, son superiores a las tensiones de transformación, tanto en las correspondientes a tracción como en compresión. Es decir, en cada ciclo de carga habrá dos transformaciones martensíticas inducidas por tensión, una en tracción y otra en compresión.

La tabla III ofrece los resultados de los ensayos de fatiga, mostrándose las siguientes magnitudes: $\sigma_{\text {máx }}=$ carga máxima aplicada, $N_{\mathrm{f}}=$ número de ciclos a fractura y $\epsilon_{\text {cum }}=$ deformación plástica acumulada.

De los ensayos de fatiga se pueden destacar los siguientes aspectos:

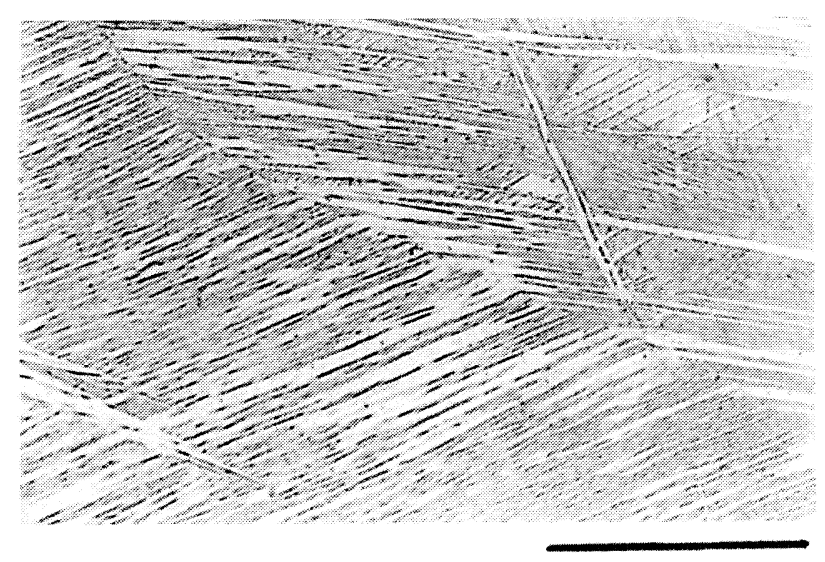

$10 \mu \mathrm{m}$

FIG. 4.- Placas martensíticas de origen térmico.

Fig. 4.-Martensite plates of thermic origen. 


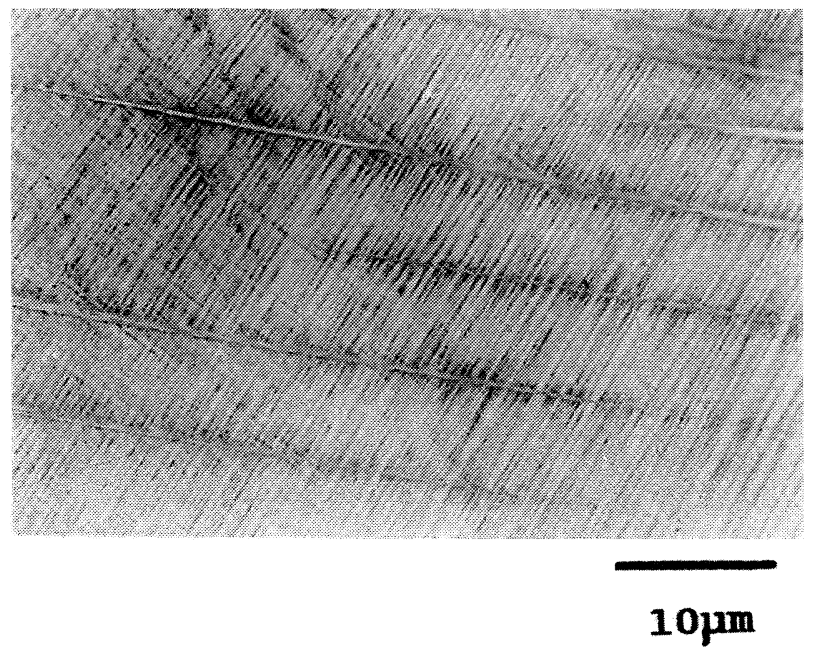

FIG. 5.- Placas martensíticas inducidas por tensión.

Fig. 5.-Stress-induced martensitic plates.

- En los registros gráficos obtenidos en el ensayo, se observa que los ciclos tensión-deformación no son simétricos, ya que se puede apreciar cómo la compresión produce a menores tensiones la transformación martensítica con respecto a la tracción, según ya se observó en los ensayos mecánicos estáticos.

- Se aprecia un endurecimiento del material a medida que aumentan los ciclos de carga. En el primer ciclo, la tensión necesaria para producir la deformación solicitada era de 138, 132 y 126 MPa para el policristal, bicristal y monocristal, respectivamente, y en el ciclo anterior a la propagación rápida de la grieta, era de $158 \mathrm{MPa}$ para el policristal, $151 \mathrm{MPa}$ para el bicristal y 142 Mpa para el monocristal.

La tasa máxima de endurecimiento es la del policristal, ya que los límites de grano favorecen la estabilización de placas de martensita que producen anclajes de las dislocaciones.

TABLA III- Resultados de los ensayos de fatiga, siendo: $\sigma_{\text {máx }}=$ carga máxima aplicada, $N_{\mathrm{f}}=$ número de ciclos a fractura y $\epsilon_{\text {cum }}=$ deformación plástica acumulada

TABLE III-Fatigue tests results. $\sigma_{\max }=$ maximum load applied, $\mathrm{N}_{f}=n^{\circ}$ of cycles to rupture and $\epsilon_{\text {cum }}=$ accumulated plastic deformation

\begin{tabular}{|l|c|r|c|}
\hline & $\sigma_{\text {máx }}, \mathrm{Mpa}$ & $N_{\mathrm{f}}$ & $\epsilon_{\text {cum }}, \mathrm{MPa}$ \\
\hline Policristal & 158 & 527 & 0,3 \\
\hline Monocristal & 142 & 23.416 & 4,2 \\
\hline $\begin{array}{l}\text { Bicristal } \\
(311) / /(110)\end{array}$ & 151 & 1.538 & 0,7 \\
\hline
\end{tabular}

- El número de ciclos hasta la rotura es muy superior en el monocristal que en las muestras con límite de grano. En estos últimos casos, la nucleación y la propagación de grieta se produce por los límites de grano, y se justifica por la gran energía interna que poseen, debido a la elevada anisotropía elástica. Las fracturas observadas eran frágiles en los policristales y en los bicristales, y tenían mayor deformación plástica en los monocristales. Este hecho se confirma al determinar los valores de deformación plástica acumulada a fractura, que no son superiores a 1 en los mono y bicristales y a 4,2 en los monocristales.

- Durante el ensayo de fatiga en el bicristal, éste se paró durante unos segundos en el ciclo $N=50$ para observar la superficie al microscopio. Se apreció que en los límites de grano ya existía martensita estabilizada, no observándose ninguna placa en el resto del material. Estas placas, en los límites de grano, fragilizan el material produciendo una disminución de la vida a fatiga.

Una vez rotas las probetas por fatiga, se cortaron muestras para determinar las temperaturas de transformación y las magnitudes termodinámicas asociadas a ellas y compararlas con las de las muestras sin ensayar. El resultado fue que ni los policristales, bicristales, ni monocristales presentaban picos de transformación martensítica al enfriar. Esta pérdida de propiedades de memoria de forma y superelasticidad, se debe a la gran cantidad de martensita estabilizada anclada en una gran maraña de dislocaciones que evita cualquier tipo de transformación.

\section{CONCLUSIONES}

En los ensayos de tracción y compresión, se observó que la tensión de transformación $B \rightarrow$ SIM es mínima para el policristal y máxima para el monocristal. Los monocristales son menos resistentes, pero mucho más dúctiles, debido a que la ausencia de límites de grano proporciona mayor movilidad a las dislocaciones y evita las incompatibilidades de la deformación que se producen en los límites de grano de los policristales. Los bicristales con el límite de grano perpendicular al eje de tensión se comportan de forma más parecida a la de los policristales que a la de los monocristales. En todos los casos, se observa la rotura intergranular. $\mathrm{Al}$ realizar el ensayo de compresión, se comprobó que las tensiones de transformación $B \rightarrow$ SIM son menores que en el ensayo de tracción; es decir, con esfuerzos compresivos es más fácil inducir martensita.

Se aprecia un endurecimiento del material a medida que aumentan los ciclos de carga. La tasa máxima de endurecimiento es la del policristal, ya 
que los límites de grano favorecen la estabilización de placas de martensita que producen anclajes de las dislocaciones.

El número de ciclos hasta la rotura preciso es muy superior en el monocristal que en las muestras con límite de grano. En estos últimos casos, la nucleación y la propagación de grieta se producen por los límites de grano y se justifica por la gran energía interna que poseen, debido a la elevada anisotropía elástica. Se aprecia, al ir ciclando el material, la aparición de martensita estabilizada, que produce una pérdida de la termoelasticidad de la aleación.

\section{Agradecimiento}

Los autores agradecen a la CICYT la concesión del proyecto de investigación MAT92-1025-C02-02 con el que se ha sufragado parcialmente este trabajo.

\section{REFERENCIAS}

(1) Miyazaki, S., OtsuKa, K., SaKamoto, H. y Shimizu, K. Trans. Jpn. Inst. Met., 22, 1981: 244.
(2) Delaey, L., Janseen, J., van de Masrelaer, D., Dullenkopf, G. y Deruyttere, A. Scr. Metall., 12, 1988: 373.

(3) Metton, K.N. y Mercier, O. Scr. Metall., 13, 1979: 73.

(4) MiYazaKI, S., OtsuKa, K. y Suzuki, Y. Scr. Metall., 15, 1981: 287.

(5) MiYaZaKi, S. y OtsuKa, K. Sci. Rep. PITU A29 Supple 1, 1981: 111 .

(6) MiYazaki, S., KawaI, T. y Otsuka, K. Journal de Physique, Colloque C4, Supplement n. 12 (43), 1982: 813-819.

(7) Takezawa, K., Izymi, T., Ciba, H. y Sato, S. Journal de Physique, Colloque C4, Supplement n. 12 (43), 1982: 804-807.

(8) SAde, M., Rapacioli, R. y Ahlers, M. Acta Metall., 33, 1985: 487.

(9) SAde, M., KumpFert, J. y Hornbogen, E. Metallkde., 79, 1988: 678 .

(10) Picornell, C., Cesari, E. y Sade, M. Materials Science Forum 56-58. Proceedings of ICONAT-89. 1990: 741.

(11) GuIllemany, J.M., Gil, F.J. y Miguel, J.R. Fundición, 7, 1987: 31-37.

(12) Gulllemany, J.M. y Gil, F.J. J. Mater. Sci., 26, 1991: 4.626-4.630.

(13) Guillemány, J.M. y Gil, F.J. Thermochim. Acta, 190, 1991: 185-189.

(14) GuIllemany, J.M. y GIL, F.J. Mater. Res. Bull., 25, 1990: 1.325-1.332.

(15) Gil, F.J. y Guilemany, J.M. Prakt. Metallogr., 28, 1991: 585-591. 